Another Look into the Central Nervous System Decussation Reveals A New Model for Evolution

*DR HOSSEIN AKBARI.MD

NEUROLOGIST,NEUROSCIENCE RESEARCHER

TEHRAN,IRAN.

Akbarih2015@gmail.com

MEHRANA FALLAHIAN,MSc

PSYCHOLOGYST

NEUROPSYCHOLGY RESEARCHER

TEHRAN IRAN

mehrebarin@yahoo.com 


\title{
Another Look into the Central Nervous System Decussation Reveals A New Model for Evolution
}

Hossein akbari, MD

Mehrana fallahian, MSc

\begin{abstract}
:
Most large fibers in central nervous system of vertebrates are decussated. The cause or the evolutionary force of this phenomenon is not convincingly described. Here we show a pattern for this nervous system property that simultaneously is found to a more basic rule in evolution.

The idea here is the "function to anatomy" theorem. We claim that functions of physical, biochemical, enzymatic, etc., in lower scale of living organisms "emerge" to anatomy in larger scales. And reversely, the anatomy we see in multicellular organisms are/were functions in microscopic scales.

We propose descriptions, predictions, and a probable road map to what this can implicate in biological and medical issues.
\end{abstract}

Keywords: CNS decussation; evolution theory; function to anatomy

\section{INTRODUCTION}

Central nervous system in vertebrates is decussated in many parts(i.e. the hemispheres of brain represent the contra lateral body in most parts ,right hemisphere rules and senses the left side of the body and vice versa. $)^{1}$

As a rule decussation is not present in invertebrates except for few instances. ${ }^{2}$

This decussation in vertebrates also predominantly occurs in tracts that modulate spatial, organized data whereas olfactory, vestibulospinal and reticulospinal systems are not decussated. ${ }^{3,4}$

This contralateral organization is a great unanswered question "why my right hand is controlled by left brain?" It is not just a simple curiosity; the answer may cover a large field in evolution mechanisms.

There are some explanations but it is actually not clear what evolutionary force made it to be so.

\section{HISTORY}

Hippocrates by his description that "convulsion seizes the other side of body" was the first to recognize this. ${ }^{5}$ In $18^{\text {th }}$ and $19^{\text {th }}$ century anatomists discovered decussating pathways. ${ }^{7}$ The first theory for explaining this was the visual map theory by Ramon y Cajal in $1898^{8}$ that decussation of optic pathways is to repair the inverted image in retina that projected to visual cortex. ${ }^{7}$ The next theory was brought by Bratenbey who argued that correcting image is not an evolutionary force for decussation but it is the taxis-kinesis behavior(e.g. smell with olfactory sense which is not crossed in nervous system and movement toward it which is crossed and this has advantages) that underlines the evolution. ${ }^{2}$ 
The other theory is that decussation is a response to the defensive coiling reflex (i.e. the withdrawing from threatening side).it is not well accepted because it is observed in invertebrates with no decussation too. ${ }^{9,10}$

Another theory is "topological constrain on 3D wiring" that shows by math that increasing the number of wiring connections pushes the system to decussate to decrease the rate of erroneous connections. ${ }^{11}$

Another theory is that for a vertebrate to escape from a potential danger there must be limb contraction opposite to the danger and limb extension in the same side of danger which correlates with motor decussation and rubrospinal pathways(extension of limbs) no decussation. ${ }^{3}$

The most accepted theories however are twist theories, one by Krisbourne ${ }^{11}$, the somatic twist theory and the other by Lussanet ${ }^{12,13}$, the axial twist theory. In axial twist theory it is proposed that a 90 degree left turn around the body axis evolved in the common ancestor of all vertebrates.(first the embryo turns to left then for retaining symmetry a further left turn in head and a compensating right left in the body occurs). In the somatic twist theory the somatic part of the body (everything behind eyes mouth, nostrils) turned on its back.

These are an abstract of main attempts to describe decussation, while it seems they are not successful in explaining the "why" question. And not also answering why it has been emerged abruptly in vertebrates.

\section{THE THEORY: IN EVERY SCALE OF EVOLUTION/CHANGE FROM THE SMALL TO THE LARGE ONE, FUNCTIONS IN THE SMALL SCALE BECOME ANATOMY/FORM IN THE LARGER SCALE.}

Let's unpack this.

Multicellular living organisms are made of organs, tissues, cells, molecules, atoms etc. This is what we mean by scale. The main scales however are cells, some molecules, atoms, etc. we omit organs and tissues because they are not independent organisms. In every organisms/system from the small scale to the large scale there is a property called EMERGENCE. It is emergence of a property in the larger scale that can not be derived or attributed to the smaller scale/or the system parts properties. For example consciousness is an emergent property of the brain (larger/higher scale) that cannot be derived from single neurons (lower/smaller scale).

In living organisms the question of how these tiny complicated creatures called cells become another complicated multicellular organisms with complex emergent properties, is an unsolved problem.

In this theory we claim that the evolution is based this pattern:

function(enzymes ,chemical reactions ,catalysts, organelles etc, i.e. transient chemical or physical changes in subcellular scales) becomes anatomy or form in multicellular organisms .In other words the anatomic features we observe in multicellular organisms are/were functions in cells, molecules etc. For example in a cell, imagine a complex chemical reaction occurs: A becomes B by means of enzyme $X$. in this case $\mathrm{X}$ is a transient unstable molecule which is degraded after some time but in the next scale $\mathrm{X}$ becomes an organ, tissue or stable matter and will do some sort of the same action by means of that in a larger organism. It is not obviously the case for all molecules ,enzymes etc in living organisms, but reversely it is true for all anatomic features of an multicellular organism to be a chemical pathway (for example) in lower scales. 
So this is how emergence works in living systems. (And probably in non living systems from strings in string theory to whole the universe!).

\section{THE DECUSSATING PROBLEM:}

Vertebrate central nervous system is the emergence of eukaryotes nucleus .it is reasonable because the main information processing center in multicellular vertebrate is brain and in the single cell scale is nucleus and if the concept of emergence is accepted it would be the right phrase.

The function of DNA in nucleus is to produce RNA which in turn produces proteins or have other function themselves (details are eliminated). We consider the process as this: DNA represents the brain, RNA represents the spinal cord, proteins represent the action, movement or effect of the organism. RNA is the transporter of data in this image that's why it represents spinal cord which actually does the same function. When one of the DNA strands produces RNA, it is transformed to its opposite strand so it is reversed. In multicelluar scale this reversing is represented in decussation. This reversing is a FUNCTION of translation between DNA and RNA which in the CNS it emerges as a decussation of existing neuronal pathways.

This simulation seems like sort of a fractal geometry (self similarity) pattern but technically it is not. Because in fractal geometry just the same shapes are reproduced in different scales, but here shapes are not reproduced, function becomes shapes, (a new geometry?).

The result is that the main rule that governs evolution is probably this: "function becomes anatomy from a lower scale to the larger one".

\section{PREDICTIONS AND EVIDENCES:}

By introducing a new theory one must be ready to answer any question, doubt, to make explanation to unexpected evidences, etc. Here I have proposed some evidences and explanations, although there are infinite ways of surveying thing by this theory. It is the first step in an unknown path.

1. Myelin: in cell scale there is splicing of the RNA that occurs in eukaryote generally and very few in prokaryote. ${ }^{14}$ The goal of splicing is to eliminate intrones which are probably unnecessary. There are some explanations for this proess. But one of the obvious results is that it increases the rate of translation. ${ }^{15}$ In this theory myelin is an emergence of splicing process.

Predictions: a) myelin or myelin producer cells in brain (i.e. oligodandrocytes) as an emergence of splicing process must have other functions beside increasing the rate of information transmission according to splicing function in cell scale. For example alternative splicing process decides one mRNA becomes this protein or that one. ${ }^{6}$ This probably happens for oligodendrocytes too (e.g. changing the transmission way, quality or something like this). These functions are not established yet.

b) Myelin is present before decussation in CNS too. So in subcellular processes, there must be a splicing-like function in the translation process before DNA is fully translated to mRNA, I haven't found it in literature.

c) Multiple sclerosis is an autoimmune myelin based abnormality. There the basic problem must be in splicing/alternative splicing in cell scales in CNS or immune system. ${ }^{16}$

2. Cerebellum: it is an anatomical feature in CNS of vertebrates with regulatory functions in movement mostly. According to the theory, there must be nucleotide based molecules in cells which regulate mRNA translation which cerebellum 
represents its function. It is probably a RNA molecule; because cerebellum fibers don't decussate and these must be noncoding RNAs. Both long noncoding RNAs and miRNAs(micro RNAs) are candidates. ${ }^{17}$ In search to understand and treat diseases of cerebellum special focus on these types of molecules will be helpful.

3. ALS: The coding mRNA and its function in this picture become motor pathways. Motor neurons in spinal cords are involved in ALS. According to the theory probably instability of the full processed mRNA is involved in ALS. ${ }^{20}$

Concise explanation is: noncoding RNAs become cerebellum and sensory pathways (these pathways are complicated in explanation since they have many interfering connections and different types of sensation and needs a complete separate paper). Motor pathways represent mRNA and splicing process becomes myelin.

ANOTHER QUESTION: why don't we observe decussation in invertebrates? Their cells are like vertebrate cells, eukaryotes, and so far there isn't much difference in invertebrates and vertebrates cells.

Our answer is based on the evolutionary scales too. In this picture the invertebrates (with an outer skeleton in some), represent functionally the prokaryotes (or better proprokaryotes), with cell wall and no inner skeleton. There is no organized CNS in invertebrates (unlike vertebrates) that correlates with prokaryotes without an organized nucleus. Vertebrate line represents the eukaryote line with inner skeleton in both and a more organized CNS.

But prokaryotes have DNA; like eukaryotes and in their function it decussates. So what is the case? The interpretation is this: there was an ancestor single cell organism before prokaryotes that had RNA as its genetic code. It seems that the invertebrate line of evolution is emergence of that missing ancestor because RNA doesn't need to be translated to its opposite strand so no decussation emerged in invertebrates. It sounds that RNA-world hypothesis is consistent with this view.

In more complicated invertebrates there is tendency toward decussation because the outer shape of CNS in these organisms becomes like vertebrates (i.e. invertebrate chordate). ${ }^{19}$ This type of invertebrate may represent the prototype prokaryotes we know.

A description: it is needed to clarify here that what we mean by scale doesn't necessarily contribute all branches of organisms. A grasshopper is not an emergence, neither is the insect line of animals. Evolution road in this theory contains the very main branches. Changes through subbranches are not considered as main emergent properties. So when we mention invertebrate we mean a prototype invertebrate. Darwinian evolution pattern is not wrong at all. It works very well in sub branches in evolution tree and also it may be at least one of the ways this function to anatomy pattern works. Our theory implicates little about the "how" question but it is the answer to the "why" question.

\section{CONCLUSION:}

In this theory we propose that evolution is not just a blind selection; no pure chance, and that there is a pattern. We derived it by trying to answer the decussation enigma in vertebrates: why my left brain controls right hand? There are a few explanations before this but none were convincing, they didn't cover all the aspects. So far it is the 
best explanation: function in lower scales becomes anatomy in higher level/scale. The scales are the levels in which independent organisms can exist. Here are for example cells then multicellular organisms. In this scaling nonliving matter like molecules and atoms and etc also are present.

Function to anatomy theorem is probably the basic pattern in evolution.

As an example we described the decussating pattern in vertebrates: it is the emergence of "DNA becomes RNA inside cells" to the" brain messages through spinal cord (DNA represents brain and RNA represents the spinal cord). So when one imagines this picture, then it would be just logical that motor pathways decussate because when DNA is translated to RNA it becomes the opposite strand of itself. This opposition shows itself as decussation.

The story above may seem to be a philosophical, metaphoric idea, but we need a new vision in biology, evolution and even medicine. I have adopted this from theoretical physics because it is very successful in putting thing forward by mixing theory and experiment and let the ideas come and then see what happens.

There exist many unexpected predictions, findings and progress behind this theory that needs an interested group of scientists biologists, evolutionarists, even physicists to explore that.

\section{References:}

1.Nieuwenhuys, R.; Donkelaar, H.J.; Nicholson, C.; Smeets, W.J.A.J.; Wicht, $\mathrm{H}$. (1998). The central nervous system of vertebrates. New York: Springer. ISBN 9783642621277.

\section{Braitenberg V. Taxis, kinesis and decussation. Prog Brain Res. 1965;17:210-222. doi:10.1016/s0079-6123(08)60163-6}

3.Vulliemoz S, Raineteau O, Jabaudon D. Reaching beyond the midline: why are human brains cross wired?. Lancet Neurol. 2005;4(2):87-99. doi:10.1016/S1474-4422(05)00990

4.ten Donkelaar HJ. Evolution of the red nucleus and rubrospinal tract. Behav Brain Res. 1988;28(1-2):9-20. doi:10.1016/0166-4328(88)90072-1

5.Breitenfeld T, Jurasic MJ, Breitenfeld D. Hippocrates: the forefather of neurology. Neurol Sci. 2014;35(9):1349-1352. doi:10.1007/s10072-014-18693

6.McManus CJ, Graveley BR. RNA structure and the mechanisms of alternative splicing. Curr Opin Genet Dev. 2011;21(4):373-379. doi:10.1016/j.gde.2011.04.001

Llinás RR. The contribution of Santiago Ramón y Cajal to functional neuroscience. Nat Rev Neurosci. 2003;4(1):77-80. doi:10.1038/nrn1011

7.Mora C, Velásquez C, Martino J. The neural pathway midline crossing theory: a historical analysis of Santiago Rámon y Cajal's contribution on 
cerebral localization and on contralateral forebrain organization. Neurosurg Focus. 2019;47(3):E10. doi:10.3171/2019.6.FOCUS19341

8. Llinás, R.R.). "The contribution of Santiago Ramón y Cajal to functional neuroscience". Nat. Rev. Neurosci. 2003; 4 (1): 77-80.

9.Kinsbourne M. Somatic twist: a model for the evolution of decussation. Neuropsychology. 2013;27(5):511-515. doi:10.1037/a0033662

10.Sarnat HB, Netsky MG: Evolution of the Nervous System. New York: Oxford University Press, 1974

11.Shinbrot T, Young W. Why decussate? Topological constraints on 3D wiring. Anat Rec (Hoboken). 2008;291(10):1278-1292. doi:10.1002/ar.20731

12.de Lussanet, Marc H.E., 2019, "Opposite asymmetries of face and trunk and of kissing and hugging, as predicted by the axial twist hypothesis" PeerJ Vol. 7, pp e7096, 2167-8359

13.de Lussanet, M..E., \& Osse, J..M. (2012). An ancestral axial twist explains the contralateral forebrain and the optic chiasm in vertebrates, Animal Biology, 62(2), 193-216.

14. Lamolle G, Musto H. Why Prokaryotes Genomes Lack Genes with Introns Processed by Spliceosomes?. J Mol Evol. 2018;86(9):611-612. doi:10.1007/s00239-018-9874-4

15.Vuong CK, Black DL, Zheng S. The neurogenetics of alternative splicing. Nat Rev Neurosci. 2016;17(5):265-281. doi:10.1038/nrn.2016.27

16. Hecker M, Rüge A, Putscher E, et al. Aberrant expression of alternative splicing variants in multiple sclerosis - A systematic review. Autoimmun Rev. 2019;18(7):721-732. doi:10.1016/j.autrev.2019.05.010

17.Serrano M. Epigenetic cerebellar diseases. Handb Clin Neurol. 2018;155:227-244. doi:10.1016/B978-0-444-64189-2.00015-9

18.Green SA, Simoes-Costa M, Bronner ME. Evolution of vertebrates as viewed from the crest. Nature. 2015;520(7548):474-482.

doi:10.1038/nature14436

19.Kozmik Z. The Amphioxus Model System. Int J Dev Biol. 2017;61(10-1112):571-574. doi:10.1387/ijdb.170332zk

20. Tank EM, Figueroa-Romero C, Hinder LM, et al. Abnormal RNA stability in amyotrophic lateral sclerosis. Nat Commun. 2018;9(1):2845. Published 2018 Jul 20. doi:10.1038/s41467-018-05049-z 
